\title{
Prioritization of rs 187728237 and rs80320514 as miRNA-related Variants of Human AEG-1 Gene
}

Semira Kheiri (PhD student)

Laboratory Sciences Research Center, Golestan University of Medical Science, Gorgan, Iran

\section{Azadeh Aliarab (MSc)}

Department of Clinical Biochemistry, School of Medicine, Shahid Beheshti University of Medical Sciences, Tehran, Iran

Hamid Haghighatfard (MSc) Cellular \& Molecular Research Center, Golestan University of Medical Sciences, Gorgan, Iran

Seyed Hossein Sadeghi (PhD student)

Department of Medical Genetics, School of Medicine, Shahid Beheshti University of Medical Sciences, Tehran, Iran

Medical Cellular \& Molecular Research Center, Golestan University of Medical Sciences, Gorgan, Iran

Corresponding author: Seyed

Hossein Sadeghi

Tel: +989190334976

E-mail: Hsadeqi86@gmail.com

Address: Department of Medical

Genetics, School of Medicine, Shahid

Beheshti University of Medical

Sciences, Tehran, Iran

Received : 28 Sep 2016

Revised: 01 Jul 2016

Accepted: 31 Jul 2016

Semira Kheiri https://orcid.org/0000-0002-8842 3985

Seyed Hossein Sadeghi 0000-0001-9599-615

\section{ABSTRACT}

Background and Objectives: 3 ' untranslated region (3'UTR) single nucleotide polymorphisms (SNPs) represent genetic variations that may potentially affect binding of miRNA to coding genes, potentially leading to complex disorders. We aimed to perform in silico analysis of the potential phenotypic effect of 3'UTR SNPs on human astrocyte elevated gene-1 (AE(6-1), a newly identified candidate cancer gene.

Methods: We gathered a list of all 3'UTR SNPs located in the human AEG-1 gene from the SNP database. Analysis of the potential effects was done using MirSNP and MicroSNiper.

Results: Analysis by the MirSNP estimated that rs187720237 might increase the affinity of two miRNAs and decrease the affinity of 10 other miRNAs to the AEG-1 transcript. Moreover, MicroSNiPer showed that rs00320514 might affect 24 putative miRNA binding sites in the 3'UTR of $A E G-1$.

Conclusion: Based on our findings, it can be concluded that the 3'UTR SNPs located in the human $A E G-1$ gene may be within the miRNA targets of the transcript, therefore affecting the stability of putative miRNA-target interactions.

Keywords: AEG-1, miRNA, SNPs, 3' Untranslated Region.

This paper should be cited as: Kheiri S, Aliarab A, Haghighatfard H, Sadeghi SH[Prioritization of rs187728237 and rs80320514 as miRNA-related Variants of Human AEG-1 Gene]. mljgoums. 2018; 12(3):6-11 


\section{INTRODUCTION}

Astrocyte elevated gene-1 (AEG-1) or 3D3/lyric is thought to be associated with several types of cancer, including hepatocellular carcinoma (1-2), breast cancer, malignant gliomas, melanomas (3-4), and neurodegeneration (5). It is also known as human metadherin and a downstream target molecule for $H$-Ras and $c-M y c$ (6). Product of the gene induces anchorage-independent growth and tumor cell invasion by increasing the expression of adhesion mediators via NF$\kappa \mathrm{B}$ pathway (6-7). Therefore, it is also considered a direct regulator of angiogenesis (3). In addition, knockdown of $A E G-1$ inhibits proliferation of human prostate cancer, neuroblastoma and melanoma cells, and induces apoptosis in prostate cancer and neuroblastoma cells (8). Accordingly, AEG-1 may be a promising target for adjuvant therapy in cancer (9).

AEG-1 mRNA encodes a 582 amino acid protein with a predicted molecular mass of 64 $\mathrm{kDa}(2,10)$, with highly conserved amino acid sequences among vertebrates (11-12). The AEG-1 gene consists of 12 exons and 11 introns located at chromosome 8q22 (13). MicroRNAs (miRNAs) are non-coding RNAs with 21-25 nucleotides that are key posttranscriptional modulators (14-16). Recent evidence show that even a single miRNA can affect hundreds of genes (17) and become involved in various cellular processes and developmental pathways (18). In addition, they can elevate or reduce expression of protein-coding genes by targeting the 3untranslated regions (3'UTRs) (19). Seed region residing in mature miRNAs is the crucial binding location for translational regulation (20). The region complementary to the miRNA seed is flexible within the miRNA and regulates mRNA function. Base pairing of the nucleotides in the seed region to complementary sites affects miRNAs length and its interaction with a specific mRNA (2123).

Single nucleotide polymorphism (SNP) is the most common genetic variation among humans. However, only a minority of SNPs could be considered as markers for underlying susceptibility to different human disorders (24). Among these alterations, SNPs in the seed region of miRNAs may generate/destroy miRNA binding sites, and affect traits and disease pathogenesis (25). Identification of allele-specific miRNA-mRNA interactions could be of great importance for gaining a better understating of the genetics of phenotypic variations. Since experimental analysis is almost impossible for screening all functionally important SNPs, a computational strategy based on biochemical changes and/or sequence information has been proposed for the systematic analysis of SNPs. There is a growing interest in predicting the miRNArelated SNPs for a disease-associated gene that are believed to have no function (26). Considering the continuing increase in frequency of SNPs in miRNA target sites (miRTSs) and lack of enough data on miRNArelated SNPs in the $A E G-1$ gene, we aimed to predict SNPs that may potentially affect the miRTSs associated with this gene.

\section{MATERIAL AND METHODS}

SNPs in the 3'UTR region of the human $A E G$ 1 gene and related mRNA accession number (NM178812) were retrieved from the National Center for Biotechnology Information (NCBI) database of SNPs (http://www.ncbi.nlm.nih.gov/projects/SNP). MicroSNiPer was used to estimate the impact of miRNA binding site SNPs on candidate genes. This online tool provides information about the generation or disruption of putative miRNA binding sites in a gene in presence of alternative SNP alleles. The tool increases flexibility at the input stage. The rs ID interface was also used in the study. MicroSNiPer can generate complementarity between the 5' end of miRNA and its 3'UTR target site, with primary focus on enhancing the specificity of finding the binding sites. In order to filter false positive predictions and capture most human miRTSs, stretches of 7 to 9 nucleotides starting from the 5 ' -end of the miRNA were selected in the analysis (16). The reliability of miRTS prediction is improved by a requirement for perfect seed pairing, especially when the seed site is conserved in the UTR regions of whole-genome alignments. We also used MirSNP, which utilizes miRanda algorithm for characterizing potential miRNArelated SNPs. All retrieved SNP IDs were searched through MirSNP to obtain prediction results. MirSNP database can also present generation or disruption energy for miRNA hybrid formation in wild-type and mutant alleles, in which a larger negative charge increases the chance of gain of seed (27). 


\section{RESULTS}

The human AEG-1 gene contained 316 SNPs in the 3'UTR region. Based on the results of MirSNP for 316 3'UTR SNPs, 66 (20.9\%) were determined to alter miRNAs binding (data shown in supplementary table 1). These variants could influence the function of AEG-1 mRNA. Compared to other 3'UTR SNPs, analysis in the MirSNP software showed that 28 SNPs may significantly increase the putative miRNAs binding sites in this gene. MirSNP also predicted 21 mutated alleles that may decrease the seed length of several miRNAs targeting the related transcripts of the gene. Our evaluations in MirSNP also showed that the rs187728237 significantly affects the miRNA seed. We also found two gain of miRTSs and ten losses of miRTSs. Moreover, no target was found for the remaining $250(79.1 \%)$ 3'UTR SNPs (Table 1). In MicroSNiPer, 67 (21.2\%) SNPs were determined to affect the miRNA seeds, which were completely different from the results obtained by MirSNP. This algorithm identified 43 variations that might absolutely disrupt the miRNA seeds from seven (and more) to zero bp, while five other variants could exert an opposite effect. Overall, MirSNP and MicroSNiPer detected 143 and 290 different miRNAs that target $A E G-1$, respectively (Table 2 ).

Table1- Analysis of rs187728237 by MirSNP

\begin{tabular}{|c|c|c|c|c|}
\hline \multicolumn{5}{|c|}{ MTDH/miRNA targets } \\
\hline \multirow[t]{19}{*}{ rsID } & mRNA & miRNA & Energy & Effect by \\
\hline & accession & & change & SNP on \\
\hline & NO(NM) & & $(\mathrm{Kcal} / \mathbf{m o l})$ & 3'UTR \\
\hline & 178812 & hsa-miR- & Wild Type: - & Gain \\
\hline & & 3609 & 15.40 & \\
\hline & & & SNP Type: - & \\
\hline & 178812 & hsa-miR- & Wild Type: - & Gain \\
\hline & & 548ah-5p & 13.60 & \\
\hline & & & SNP Type: - & \\
\hline & & & 14.60 & \\
\hline & 178812 & hsa-miR-559 & Wild Type: - & Loss \\
\hline & & & 17.50 & \\
\hline & & & SNP Type: & \\
\hline & & & 0.00 & \\
\hline & 178812 & hsa-miR- & Wild Type: - & Loss \\
\hline & & $548 b-5 p$ & 17.30 & \\
\hline & & & SNP Type: & \\
\hline & & & $\mathbf{0 . 0 0}$ & \\
\hline & 178812 & hsa-miR- & Wild Type: - & Loss \\
\hline \multirow{30}{*}{$\begin{array}{l}\text { rs187728237 } \\
\text { U --> C }\end{array}$} & & & $\begin{array}{l}15.00 \\
\text { SNP Type: }\end{array}$ & \\
\hline & & & 0.00 & \\
\hline & 178812 & hsa-miR- & Wild Type: - & Loss \\
\hline & & $548 d-5 p$ & 19.40 & \\
\hline & & & SNP Type: & \\
\hline & & & $\mathbf{0 . 0 0}$ & \\
\hline & 178812 & hsa-miR- & Wild Type: - & Loss \\
\hline & & $548 h-5 p$ & 14.60 & \\
\hline & & & SNP Type: & \\
\hline & & & $\mathbf{0 . 0 0}$ & \\
\hline & 178812 & hsa miR- & Wild Type: - & Loss \\
\hline & & $548 w$ & 14.90 & \\
\hline & & & SNP Type: & \\
\hline & & & $\mathbf{0 . 0 0}$ & \\
\hline & 178812 & hsa-miR- & Wild Type: - & Loss \\
\hline & & $5480-5 p$ & 15.60 & \\
\hline & & & SNP Type: & \\
\hline & & & 0.00 & \\
\hline & 178812 & hsa-miR- & Wild Type: - & Loss \\
\hline & & 548ab & 17.50 & \\
\hline & & & SNP Type: & \\
\hline & & & 0.00 & \\
\hline & 178812 & hsa-miR- & Wild Type: - & Loss \\
\hline & & 548am-5p & 15.60 & \\
\hline & & & SNP Type: & \\
\hline & & & 0.00 & \\
\hline & 178812 & hsa-miR- & Wild Type: - & Loss \\
\hline & & 548ay-5p & 18.60 & \\
\hline & & & SNP Type: & \\
\hline & & & 0.00 & \\
\hline
\end{tabular}




\begin{tabular}{ll}
\hline $\begin{array}{l}\text { rs80320514 [ } \\
\text { C/T] }\end{array}$ & \\
\hline Seed length & MicroRNA sets \\
& \\
& hsa-miR-1321, hsa-miR-3151, hsa-miR-3689c, hsa-miR-3689a-3p, hsa- \\
7-mer & miR-3689b-3p, hsa-miR-4665-5p, hsa-miR-608, hsa-miR-4260, hsa-miR- \\
& $202-3 p$, hsa-miR-570-5p, hsa-miR-3175, hsa-miR-30c-2-3p, hsa-miR- \\
& $548 a i$, hsa-miR-92a-2-5p, hsa-let-7d-5p \\
8-mer & hsa-miR-4447, hsa-miR-4472, hsa-miR-5698, hsa-miR-92a-2-5p, hsa-miR- \\
& $4787-5 p$, hsa-miR-4723-5p, hsa-miR-4787-5p \\
9-mer & hsa-miR-1275, hsa-miR-30c-1-3p \\
\hline
\end{tabular}

\section{DISCUSSION}

It is demonstrated that miRNAs, as posttranscriptional regulators, usually play a fundamental role in translation repression and target RNA destabilization by binding to the 3'UTR, 5'UTR or the coding region of the transcripts (28). Recent studies indicated that binding of miRNA to the 3'UTR region of transcripts is more effective in gene expression regulation (29). In addition, it has been suggested that miRNAs might interfere with numerous developmental processes, and could be considered as molecular biomarkers for several disorders (28).

Since preclinical studies in cancer biology are time consuming and extremely expensive, and require in vitro and in vivo animal experiments (30), in silico approaches prior to laboratory studies have been widely considered in recent investigations. Overexpression of the human $A E G-1$ gene has been shown in malignant gliomas, breast carcinomas and melanomas (31), and we were able to identify putative miRTSs and affinity of candidate miRNAs in the presence of 3'UTR SNPs of this gene using the MicroSNiPer and MirSNP databases. Analysis by MirSNP indicted that rs187728237 might increase the affinity of two miRNAs and decrease the affinity of 10 other miRNAs to AEG-1 transcript. This variant might also increase the affinity of hsa-miR3609 (energy charge in wild-type allele: 15.40 Kcal vs. mutant allele: $16.30 \mathrm{Kcal}$ ) and hsamiR-548ah-5p (energy charge in wild-type allele: $13.60 \mathrm{Kcal}$ vs. mutant allele: 14.60 Kcal) (Table 1). Some studies reported overexpression of hsa-miR-3609 under endoplasmic reticulum stress (32) and in lung cancer (33). Considering the predicted target mRNAs of hsa-miR-548, functional enrichment analysis showed that the
miRNAgene family plays a major role in various biological processes and human diseases such as colorectal cancer, glioma and Alzheimer's disease (34). We found that rs187728237 might reduce the affinity of other miRNA variants including hsa-miR-559 and hsa-miR-548b-5p to the $A E G-1$ gene transcript. It has been claimed that hsa-miR$548 \mathrm{~b}-5 \mathrm{p}$ is significantly decreased in squamous cell carcinoma of the tongue (35). Transcriptional and post-transcriptional mechanisms are involved in regulation of ERBB2 protein expression. Hong Chen et al. claimed that 3'UTR of the ERBB2 gene is the binding site for hsa-miR-559, indicating the possible involvement of this miRNA in $E R B B 2$ gene expression (36). Analysis of the 3'UTR variants of $A E G-1$ gene by MicroSNiPer showed that rs80320514 might affect the binding site of 24 miRNAs (Table 2), including hsa-miR-1275 and hsa-miR1321, which are thought to be involved in multiple sclerosis and pancreatic cancer, respectively $(37,38)$.

There is no direct approach for evaluating the accuracy of the predictions made by MirSNP and MicroSNiper, since the algorithms might have used different data sets. Although the predictions made by these tools were inconsistent for this subset of mutants, these AEG-1 variants should still be regarded as candidates for SNP screening.

It is suggested to conduct molecular and experimental studies to confirm our results, and further evaluate the possible regulatory functions of our predicted miRNA-related variants.

\section{CONCLUSION}

Based on our findings, it can be concluded 
that the 3'UTR SNPs located in the human AEG-1 gene may be within the miRTSs of the transcript, affecting the stability of putative miRNA-target interactions.

\section{ACKNOWLEDGMENTS}

This study has been approved and

\section{REFERENCES}

1. Yoo BK, Chen D, Su Z-z, Gredler R, Yoo J, Shah K, et al. Molecular mechanism of chemoresistance by astrocyte elevated gene-1. Cancer research. 2010; 70(8): 3249-58. doi: 10.1158/0008-5472.CAN-09-4009.

2. Yoo BK, Emdad L, Su Z-z, Villanueva A, Chiang DY, Mukhopadhyay ND, et al. Astrocyte elevated gene-1 regulates hepatocellular carcinoma development and progression. The Journal of clinical investigation. 2009; 119(3): 465-77. doi: 10.1172/JCI36460.

3. Emdad L, Lee S-G, Su ZZ, Jeon HY, Boukerche H, Sarkar D, et al. Astrocyte elevated gene-1 (AEG-

1) functions as an oncogene and regulates angiogenesis. Proceedings of the National Academy of

Sciences. 2009; 106(50): 21300-5. doi: 10.1073/pnas.0910936106.

4. Yu C, Chen K, Zheng H, Guo X, Jia W, Li M, et al. Overexpression of astrocyte elevated gene-1(AEG-1) is associated with esophageal squamous cell carcinoma (ESCC) progression and pathogenesis. Carcinogenesis. 2009; 30(5): 894-901. doi: 10.1093/carcin/bgp064.

5. Yoo BK, Emdad L, Lee S-G, Su Z-z, Santhekadur P, Chen D, et al. Astrocyte elevated gene-1 (AEG-1): A multifunctional regulator of normal and abnormal physiology. Pharmacology \& therapeutics. 2011; 130(1): $1-8$.

6. Lee S-G, Su Z-Z, Emdad L, Sarkar D, Fisher PB. Astrocyte elevated gene-1 (AEG-1) is a target gene of oncogenic Ha-ras requiring phosphatidylinositol 3kinase and c-Myc. Proceedings of the National

Academy of Sciences. 2006; 103(46): 17390-5. DOI:10.1073/pnas.0608386103.

7. Emdad L, Sarkar D, Su Z-z, Randolph A, Boukerche $\mathrm{H}$, Valerie $\mathrm{K}$, et al. Activation of the nuclear factor $\kappa B$ pathway by astrocyte elevated gene-1: implications for tumor progression and metastasis. Cancer research. 2006; 66(3): 1509-16. DOI:10.1158/0008-5472.CAN-053029.

8. Ramensky V, Bork P, Sunyaev S. Human nonsynonymous SNPs: server and survey. Nucleic acids research. 2002; 30(17): 3894-900.

9. Liu H, Song X, Liu C, Xie L, Wei L, Sun R. Knockdown of astrocyte elevated gene-1 inhibits proliferation and enhancing chemo-sensitivity to cisplatin or doxorubicin in neuroblastoma cells. Journal of Experimental \& Clinical Cancer Research. 2009; 28(1): 19. doi: 10.1186/1756-9966-28-19.

10. Li J, Zhang N, Song L-B, Liao W-T, Jiang L-L, Gong $\mathrm{L}-\mathrm{Y}$, et al. Astrocyte elevated gene-1 is a novel prognostic marker for breast cancer progression and overall patient survival. Clinical Cancer Research. 2008; 14(11): 3319-26. supported by the Golestan University of Medical Sciences, Iran. We would like to thank Mr. Esmaeil Samadian for his useful comments.

\section{CONFLICT OF INTEREST}

There is no conflict of interest to declare.

11. Sutherland HG, Lam YW, Briers S, Lamond AI, Bickmore WA. 3D3/lyric: a novel transmembrane protein of the endoplasmic reticulum and nuclear envelope, which is also present in the nucleolus. Experimental cell research. 2004; 294(1): 94-105.

12. Ying $\mathrm{Z}, \mathrm{Li} \mathrm{J}, \mathrm{Li} \mathrm{M}$. Astrocyte elevated gene 1 : biological functions and molecular mechanism in cancer and beyond. Cell \& bioscience. 2011;1(1): 36. doi: 10.1186/2045-3701-1-36.

13. Lee S-G, Kang D-C, DeSalle R, Sarkar D, Fisher PB. AEG-1/MTDH/LYRIC, the beginning: initial

cloning, structure, expression profile, and regulation of expression. Advances in cancer research.

2013;120: :1-38. doi: 10.1016/B978-0-12-4016767.00001-2.

14. Lin $\mathrm{P}, \mathrm{Yu} \mathrm{S}$, Yang P. MicroRNA in lung cancer. British journal of cancer. 2010; 103(8): 1144-8. doi:10.1038/sj.bjc.6605901.

15. Hsu S-D, Lin F-M, Wu W-Y, Liang C, Huang W-C, Chan W-L, et al. miRTarBase: a database curates experimentally validated microRNA-target interactions. Nucleic acids research. 2010 39(Database issue): D1639. doi: 10.1093/nar/gkq1107.

16. Barenboim M, Zoltick BJ, Guo Y, Weinberger DR. MicroSNiPer: a web tool for prediction of SNP

effects on putative microRNA targets. Human mutation. 2010; 31(11):1223-32. doi: 10.1002/humu.21349.

17. Bartel DP. MicroRNAs: target recognition and regulatory functions. Cell. 2009; 136(2): 215-33. doi: 10.1016/j.cell.2009.01.002.

18. Kunej T, Godnic I, Horvat S, Zorc M, Calin GA. Cross talk between microRNA and coding cancer genes. Cancer journal (Sudbury, Mass). 2012;18(3):22331. doi: 10.1097/PPO.0b013e318258b771.

19. Eder M, Scherr M. MicroRNA and lung cancer. N Engl J Med. 2005; 352(23): 2446-8. DOI:10.1056/NEJMcibr051201.

20. Jevsinek Skok D, Godnic I, Zorc M, Horvat S, Dovc $\mathrm{P}$, Kovac M, et al. Genome-wide in silico screening for microRNA genetic variability in livestock species. Animal genetics. 2013; 44(6): 669-77. doi: 10.1111/age.12072.

21. Lewis BP, Burge CB, Bartel DP. Conserved seed pairing, often flanked by adenosines, indicates that thousands of human genes are microRNA targets. Cell. 2005; 120(1): 15-20.

22. Nahvi A, Shoemaker CJ, Green R. An expanded seed sequence definition accounts for full regulation of the hid 3'UTR by bantam miRNA. RNA. 2009; 15(5): 814-22. 
23. Sun G, Yan J, Noltner K, Feng J, Li H, Sarkis DA, et al. SNPs in human miRNA genes affect

biogenesis and function. Rna. 2009; 15(9):1640-51. doi: 10.1261/rna.1560209.

24. Wang C, Yang Q. Astrocyte elevated gene-1 and breast cancer (Review). Oncology letters. 2011; 2(3): 399-405.

25. Nicoloso MS, Sun H, Spizzo R, Kim H, Wickramasinghe P, Shimizu M, et al. Single-nucleotide polymorphisms inside microRNA target sites influence tumor susceptibility. Cancer research. 2010; 70(7): 278998.

26. Naderi M, Gharaei R, Soleymani-Nejadian E, Samadian E. In Silico survey of functional coding variants in human AEG-1 gene. Egyptian Journal of Medical Human Genetics. 2013; 14(4): 419-22.

27. Liu C, Zhang F, Li T, Lu M, Wang L, Yue W, et al. MirSNP, a database of polymorphisms altering miRNA target sites, identifies miRNA-related SNPs in GWAS SNPs and eQTLs. BMC genomics. 2012;13(1): 661. doi: 10.1186/1471-2164-13-661.

28. Hajjari M, Mowla SJ, Faghihi MA. Editorial: Molecular Function and Regulation of Non-coding RNAs in Multifactorial Diseases. Frontiers in genetics. 2016;7.

29. Fang Z, Rajewsky N. The impact of miRNA target sites in coding sequences and in 3' UTRs. PloS one. 2011 ; 6(3): e18067.

30. Trisilowati, Mallet DG. In silico experimental modeling of cancer treatment. ISRN oncology. 2012;2012:828701. doi: 10.5402/2012/828701.

31. Kang D-c, Su Z-z, Sarkar D, Emdad L, Volsky DJ, Fisher PB. Cloning and characterization of HIV-1inducible astrocyte elevated gene-1, AEG-1. Gene. 2005; 353(1): 8-15.
32. Dai L, Huang C, Chen L, Shan G, Li Z. Altered expression of microRNAs in the response to ER stress. Science Bulletin. 2015; 60(2): 202-9.

33. Ma J, Mannoor K, Gao L, Tan A, Guarnera MA, Zhan $\mathrm{M}$, et al. Characterization of microRNA transcriptome in lung cancer by next-generation deep sequencing. Molecular oncology. 2014; 8(7): 1208-19. doi: 10.1016/j.molonc.2014.03.019.

34. Liang T, Guo L, Liu C. Genome-wide analysis of mir-548 gene family reveals evolutionary and functional implications. BioMed Research International. 2012; 2012 :679563. doi: 10.1155/2012/679563.

35. Rentoft M, Fahlén J, Coates P, Laurell G, Sjöström $\mathrm{B}$, Rydén $\mathrm{P}$, et al. miRNA analysis of formalinfixed squamous cell carcinomas of the tongue is affected by age of the samples. International journal of oncology. 2011; 38(1): 61-9.

36. Chen H, guo Sun J, Cao X-w, Ma X-g, Xu J-p, Luo $\mathrm{F}-\mathrm{k}$, et al. Preliminary validation of ERBB2 expression regulated by $m i R-548 d-3 p$ and $m i R-559$. Biochemical and biophysical research communications. 2009; 385(4): 596-600. doi: 10.1016/j.bbrc.2009.05.113.

37. Keller A, Leidinger P, Lange J, Borries A, Schroers $\mathrm{H}$, Scheffler $\mathrm{M}$, et al. Multiple sclerosis: microRNA expression profiles accurately differentiate patients with relapsing-remitting disease from healthy controls. PloS one. 2009; 4(10): e7440. doi: 10.1371/journal.pone.0007440.

38. Ali S, Almhanna K, Chen W, Philip PA, Sarkar FH. Differentially expressed miRNAs in the plasma may provide a molecular signature for aggressive pancreatic cancer. Am J Transl Res. 2010; 3(1): 28-47. 